Supporting Information for

\title{
Magnetic and Optical Bistability Driven by Thermally- and Photo- Induced Intramolecular Electron Transfer in a Molecular Cobalt-Iron Prussian Blue Analogue
}

\author{
Dongfeng Li, ${ }^{1}$ Rodolphe Clérac, ${ }^{2 *}$ Olivier Roubeau, ${ }^{2}$ Etienne Harté ${ }^{2}$ Corine Mathonière, ${ }^{3 *}$ \\ Rémy Le Bris, ${ }^{3}$ and Stephen M. Holmes ${ }^{1 *}$ \\ ${ }^{1}$ Department of Chemistry, University of Kentucky, Lexington, Kentucky 40506-0055, USA \\ ${ }^{2}$ Université Bordeaux 1; CNRS, Centre de Recherche Paul Pascal-UPR8641, 115 avenue du Dr. A. \\ Schweitzer, 33600 Pessac, France \\ ${ }^{3}$ Université Bordeaux 1; CNRS, Institut de Chimie de la Matière Condensée de Bordeaux, UPR9048, 87 \\ avenue du Dr. A. Schweitzer, 33608 Pessac, France
}

Figure S1. Left: DSC thermograms of 1 and evolution of DSC peak as a

function of scan rate (right).

Figure S2. Simulated X-ray powder patterns of 1 at $260 \mathrm{~K}$,

$90 \mathrm{~K}$ (slowly cooled), and $90 \mathrm{~K}$ (rapidly cooled).

Figure S3. Room temperature UV-vis solution spectrum of 1 in $\mathrm{CH}_{3} \mathrm{CN}\left[C=8.58 \times 10^{-5} \mathrm{M}\right] \ldots . \mathrm{S} 3$

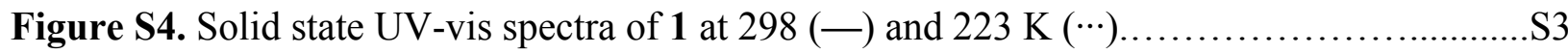

Figure S5. $\chi$ vs $T$ data obtained for 1 (where $\chi=M / H ; \Delta T=0.4 \mathrm{~K} \mathrm{~min}^{-1}$ ) between

1.8 and $300 \mathrm{~K}$ before $(\bullet)$ and after $(\bullet)$ after quenching from 300 to $5 \mathrm{~K}\left(H_{\mathrm{dc}}=1 \mathrm{~T}\right) \ldots \ldots \ldots . . . \mathrm{S} 4$

Figure S6. Left: $\chi$ vs $T$ data for 1 between 1.8 and $40 \mathrm{~K}$ and Right: $M$ vs $H$

data at $1.82 \mathrm{~K}(\bullet)$ before irradiation or quench, $(\bullet)$ after irradiation $\left[575(40) \mathrm{mW} \mathrm{cm}^{-2}\right.$ ]

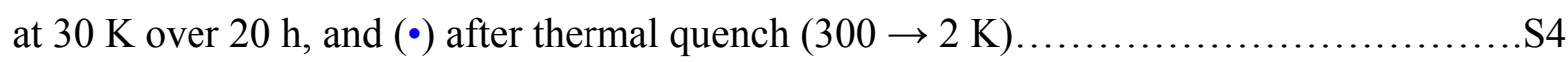

Figure S7. $\chi T$ vs time data obtained for 1 at $30 \mathrm{~K}\left(H_{\mathrm{dc}}=1 \mathrm{~T}\right)$ under

halogen white light irradiation [power $=575(40) \mathrm{mW} \mathrm{cm}^{-2}$ ].

Figure S8. $\chi T$ vs time data obtained for thermally quenched samples

$(300 \rightarrow 100 \mathrm{~K})$ of $\mathbf{1}$ at an applied static magnetic field of $H_{\mathrm{dc}}=1 \mathrm{~T}$.

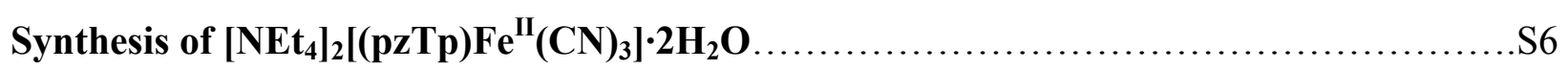

Table S1. Crystallographic data for $\left[\mathrm{NEt}_{4}\right]_{2}\left[(\mathrm{pzTp}) \mathrm{Fe}^{\mathrm{II}}(\mathrm{CN})_{3}\right] \cdot 2 \mathrm{H}_{2} \mathrm{O} \ldots \ldots \ldots \ldots \ldots \ldots \ldots \ldots \ldots . . . . . . \ldots \ldots$

Table S2. Bond Distances $(\AA)$ and angles $\left(^{\circ}\right)$ for $\left[\mathrm{NEt}_{4}\right]_{2}\left[(\mathrm{pzTp}) \mathrm{Fe}^{\mathrm{II}}(\mathrm{CN})_{3}\right] \cdot 2 \mathrm{H}_{2} \mathrm{O} \ldots \ldots \ldots \ldots \ldots . . . . \mathrm{S} 7$

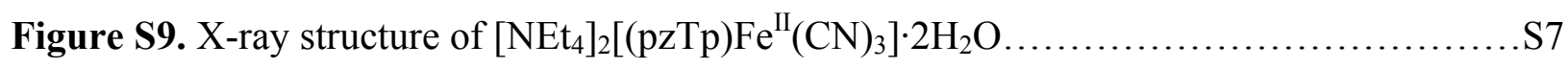



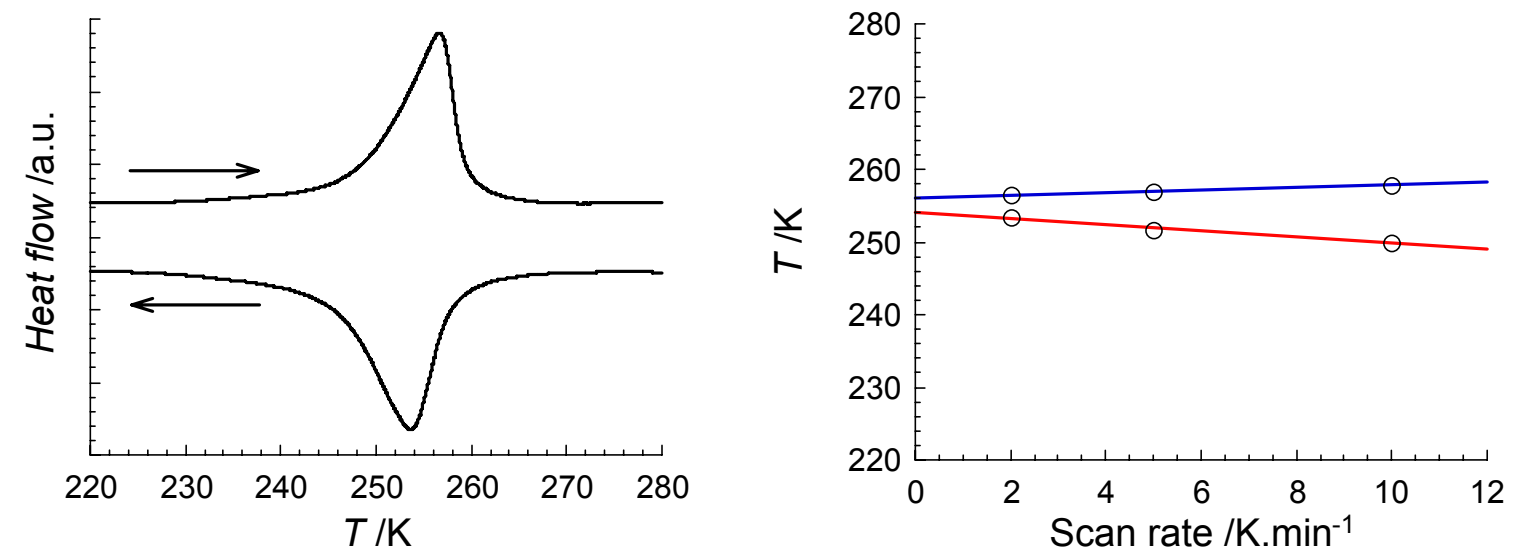

Figure S1. Left: DSC thermograms at a $2 \mathrm{~K} \mathrm{~min}^{-1}$ scan rate for $\mathbf{1}$ (endothermic peaks are pointing up) Right: Evolution of DSC peak temperature vs scan rate emphasizing tiny hysteresis effect $(c a .2 \mathrm{~K})$ near transition temperature $(255 \mathrm{~K})$. These results are in agreement with magnetic measurements suggesting negligible thermal hysteresis behavior.
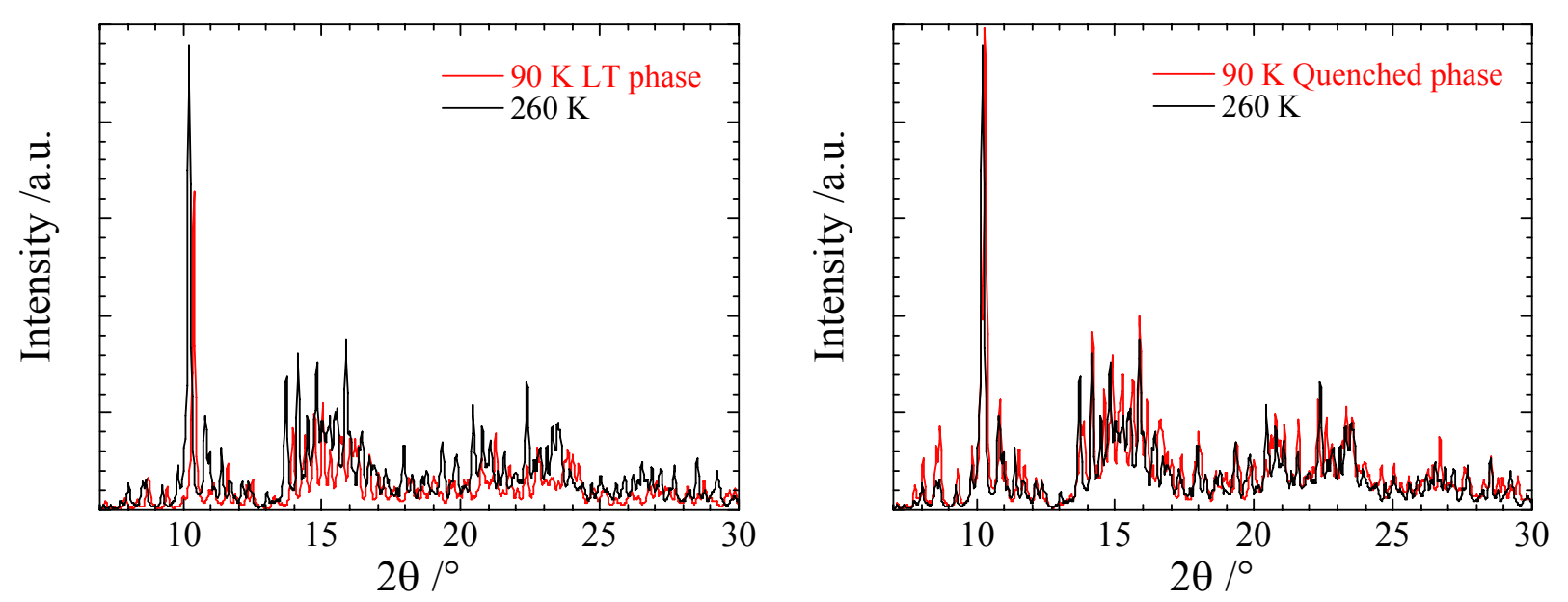

Figure S2. (left) Simulated X-ray powder patterns of 1 using the single crystal X-ray data collected at $260 \mathrm{~K}$ and $90 \mathrm{~K}$ (slowly cooled low temperature, LT, phase). Note: Comparing the $260 \mathrm{~K}$ and $90 \mathrm{~K}$ LT phase emphasize the differences between the two (left). (right) Simulated Xray powder patterns for the $90 \mathrm{~K}$ (rapidly cooled, quenched Q, phase) and high temperature (HT phase, $260 \mathrm{~K}$ ) data collected for 1 Note: Comparing the $260 \mathrm{~K}$ and $90 \mathrm{~K}$ quenched phases emphasize the similarities of both (right). 


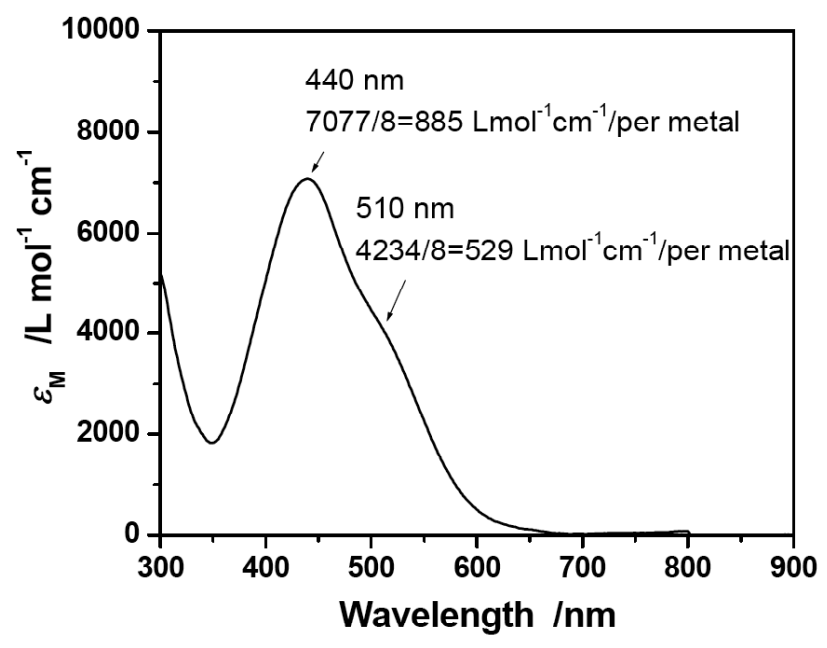

Figure S3. Room temperature UV-vis solution spectrum of $\mathbf{1}$ in $\mathrm{CH}_{3} \mathrm{CN}\left[\mathrm{C}=8.58 \times 10^{-5} \mathrm{M}\right]$.

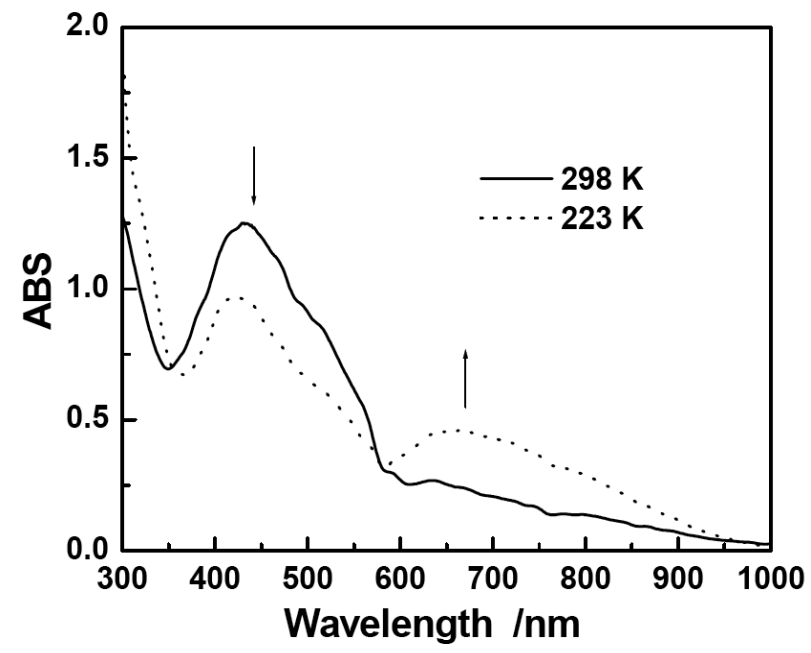

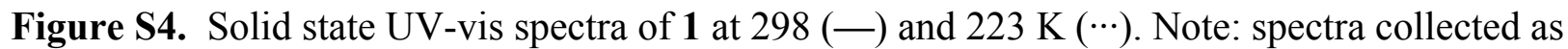
Nujol mulls between $\mathrm{KBr}$ plates. 


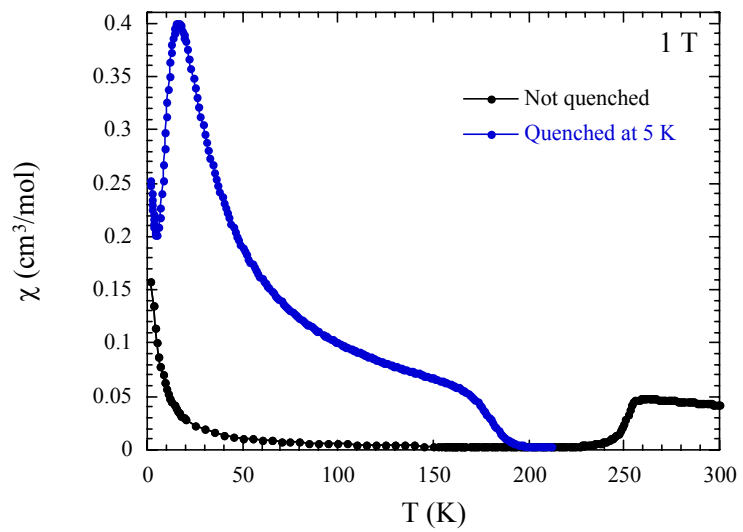

Figure S5. $\chi$ vs $T$ data obtained (where $\chi=M / H$ ) when temperature is altered at rate of $0.4 \mathrm{~K}$ $\min ^{-1}$ for 1 between 1.8 and $300 \mathrm{~K}\left(H_{\mathrm{dc}}=1 \mathrm{~T}\right)$ before $(\bullet)$ and after $(\bullet)$ rapid thermal quenching from 300 to $5 \mathrm{~K}$. The lines are guides for the eye.
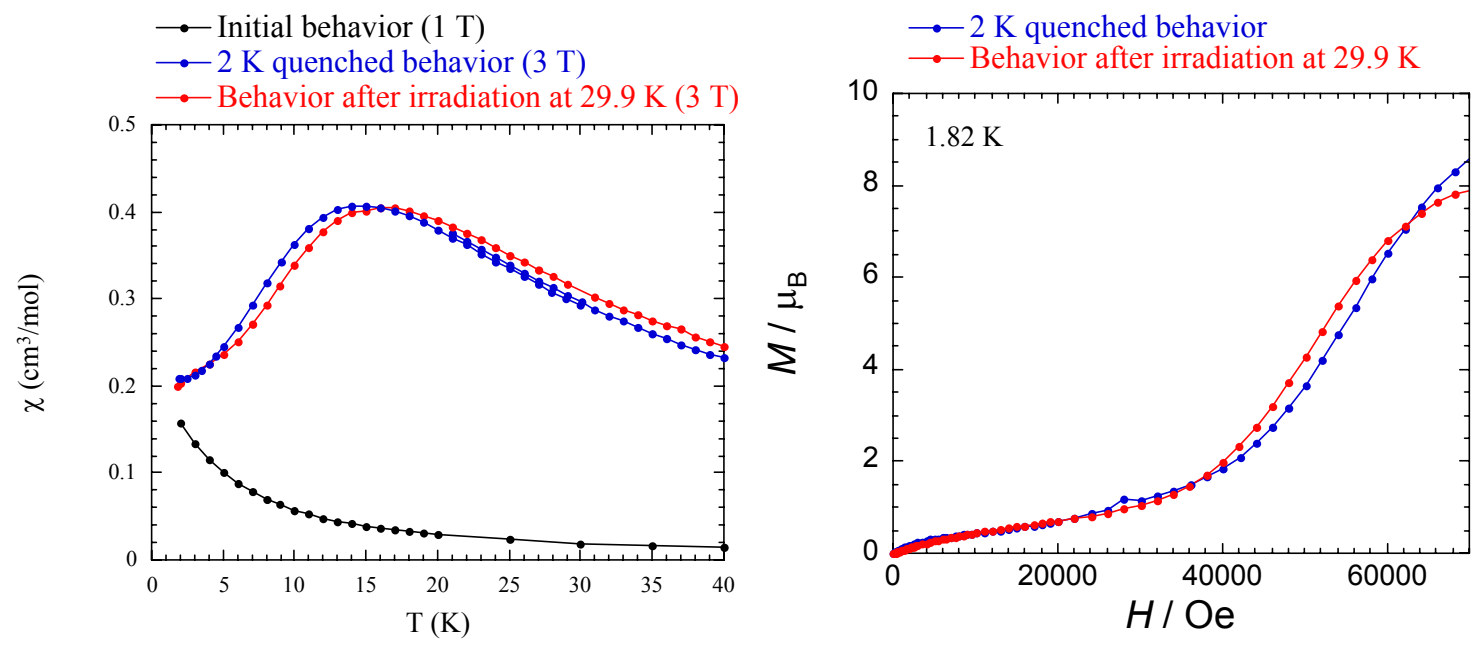

Figure S6. Left: $\chi$ vs $T$ data for 1 between 1.8 and $40 \mathrm{~K}$ and Right: $M$ vs $H$ data at $1.82 \mathrm{~K}(\bullet)$ before irradiation or quench, $\left({ }^{\circ}\right)$ after halogen white light irradiation $\left[575(40) \mathrm{mW} \mathrm{cm}^{-2}\right]$ at $30 \mathrm{~K}$ over $20 \mathrm{~h}$, and $(\bullet)$ after a thermal quench $(300 \rightarrow 2 \mathrm{~K})$. The lines are guides for the eye. Note the similarity between the photo-induced and quenched states. 


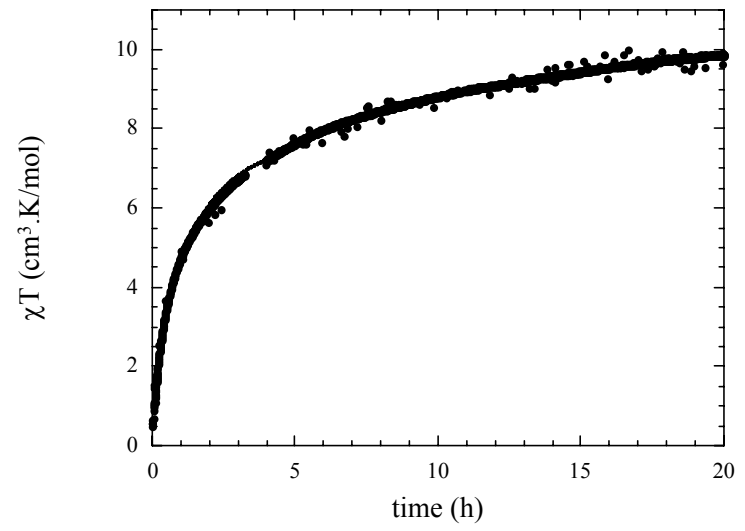

Figure S7. $\chi T$ vs time data obtained for 1 at $30 \mathrm{~K}\left(H_{\mathrm{dc}}=1 \mathrm{~T}\right)$ at an incident halogen white light power of $575(40) \mathrm{mW} \mathrm{cm}^{-2}$.

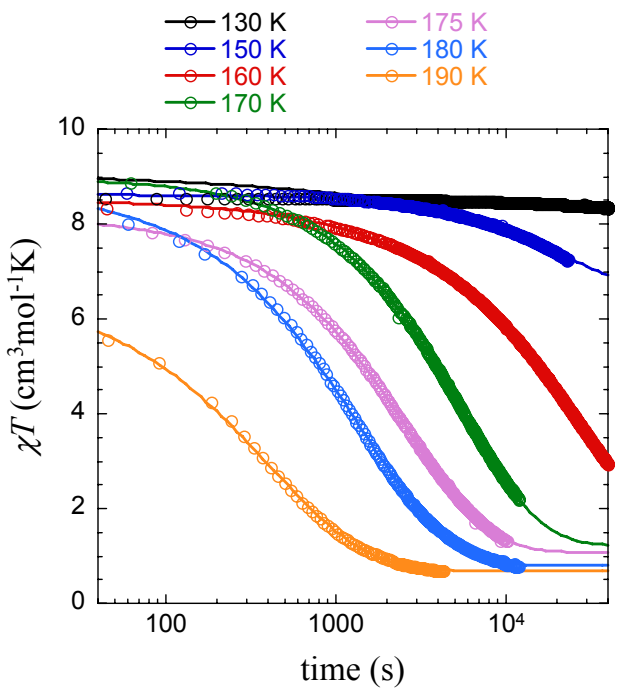

Figure S8. $\chi T$ vs time data obtained for thermally quenched samples $(300 \rightarrow 100 \mathrm{~K})$ of $\mathbf{1}$ at an applied static magnetic field of $H_{\mathrm{dc}}=1 \mathrm{~T}$. The relaxation times were deduced from the fitting the data using a stretched exponential law (solid lines). 
Synthesis of $\left[\mathbf{N E t}_{4}\right]_{2}\left[(\mathbf{p z T p}) \mathrm{Fe}^{\mathrm{II}}(\mathbf{C N})_{3}\right]$. Treatment of $\left[(\mathrm{pzTp})_{2} \mathrm{Fe}^{\mathrm{II}}\right](1.228 \mathrm{~g}, 2.00 \mathrm{mmol})$ $($ pzTp $=$ tetra(pyrazolyl)borate $)$ with $\left[\mathrm{NEt}_{4}\right] \mathrm{CN}(0.967 \mathrm{~g}, 6.20 \mathrm{mmol})$ in $\mathrm{MeCN}(40 \mathrm{ml})$ for $1 \mathrm{~h}$ at $50{ }^{\circ} \mathrm{C}$ afforded an orange solution that was evacuated to dryness. The orange residue was dissolved into $\mathrm{MeCN}(15 \mathrm{~mL})$, filtered, and the filtrate was layered with $\mathrm{Et}_{2} \mathrm{O}(40 \mathrm{~mL})$. Orange blocks were obtained after $3 \mathrm{~d}$ and were isolated via filtration, washed with $\mathrm{Et}_{2} \mathrm{O}(2 \times 5 \mathrm{~mL})$, and dried under vacuum for $2 \mathrm{~h}$. Yield: $1.22 \mathrm{~g}(86.0 \%)$. Anal Calcd. For $\mathrm{C}_{31} \mathrm{H}_{52} \mathrm{~N}_{13} \mathrm{BFe}$ C, 55.23; H, 7.80; N, 27.02 . Found: C, 56.14; H, 8.41; N, 28.49. IR (Nujol, cm ${ }^{-1}$ ): 3658 (w), 3351 (vs, br), 3240 (vs, br) 3089 (s), 2954 (vs), 2924 (vs), 2855 (vs), 2069 (vs), 2050 (vs), 1499 (vs), 1481 (vs), 1461 (vs), 1411 (vs), 1390 (vs), 1293 (vs), 1213 (vs), 1189 (s), 1174 (s), 1091 (vs), 1056 (s), 1047 (s), 1004 (s), 920 (m), 871 (m), 842 (s), 814 (s), 795 (vs), 780 (vs), 759 (vs), 669 (s), $623(\mathrm{~s}) \cdot \mu_{\mathrm{eff}}\left(\mu_{\mathrm{B}}\right)=0$.

Table S1. Crystallographic data for $\left[\mathrm{NEt}_{4}\right]_{2}\left[(\mathrm{pzTp}) \mathrm{Fe}^{\mathrm{II}}(\mathrm{CN})_{3}\right] \cdot 2 \mathrm{H}_{2} \mathrm{O}$.

\begin{tabular}{|l|l|}
\hline crystal color & brick red \\
\hline formula & $\mathrm{C}_{31} \mathrm{H}_{56} \mathrm{BFeN}_{13} \mathrm{O}_{2}$ \\
\hline formula wt & 709.55 \\
\hline crystal system & Orthorhombic \\
\hline space group & $P 2_{1} 2_{1} 2_{1}$ \\
\hline wavelength, $\lambda$ & 0.71073 \\
\hline Temperature, $\mathrm{K}$ & $90.0(2)$ \\
\hline$a, \AA$ & $14.6607(4)$ \\
\hline$b, \AA$ & $15.5088(4)$ \\
\hline$c, \AA$ & $16.0048(5)$ \\
\hline$\alpha,{ }^{\circ}$ & 90.0 \\
\hline$\beta,^{\circ}$ & 90.0 \\
\hline$\gamma{ }^{\mathrm{o}}$ & 90.0 \\
\hline$V, \AA^{3}$ & $3639.0(2)$ \\
\hline$D_{\mathrm{c}}, \mathrm{g} \mathrm{cm}{ }^{-3}$ & 1.295 \\
\hline$Z$ & 4 \\
\hline$\mu, \mathrm{mm}^{-1}$ & 0.463 \\
\hline$R_{1}{ }^{\mathrm{a}}$ & 0.0573 \\
\hline${ }_{\mathrm{w}}{ }_{2}^{\mathrm{a}}$ & 0.1010 \\
\hline${ }^{a} I>2 \sigma(I), R=\sum|| F_{\mathrm{o}}|-| F_{\mathrm{o}} \| \sum\left|F_{\mathrm{o}}\right| . R_{\mathrm{W}}=\left[\left(\sum w\left(\left|F_{\mathrm{o}}\right|-\left|F_{\mathrm{o}}\right|\right)^{2} / \sum w F_{\mathrm{o}}{ }^{2}\right)\right]^{1 / 2}$ \\
\hline
\end{tabular}


Table S2. Selected Bond Distances $(\AA)$ and Angles $\left(^{\circ}\right)$ for $\left[\mathrm{NEt}_{4}\right]_{2}\left[(\operatorname{pzTp}) \mathrm{Fe}^{\mathrm{II}}(\mathrm{CN})_{3}\right] \cdot 2 \mathrm{H}_{2} \mathrm{O}$.

\begin{tabular}{|l|l|l|l|}
\hline Fe1-C13 & $1.901(5)$ & C13-Fe1-C14 & $88.2(2)$ \\
\hline Fe1-C14 & $1.908(5)$ & C13-Fe1-C15 & $88.6(2)$ \\
\hline Fe1-C15 & $1.901(5)$ & C14-Fe1-C15 & $90.5(2)$ \\
\hline Fe1-N1 & $2.023(4)$ & C13-Fe1-N1 & $90.3(2)$ \\
\hline Fe1-N3 & $1.995(4)$ & N1-Fe1-N3 & $86.0(2)$ \\
\hline Fe1-N5 & $1.981(3)$ & N1-Fe1-N5 & $87.2(2)$ \\
\hline
\end{tabular}

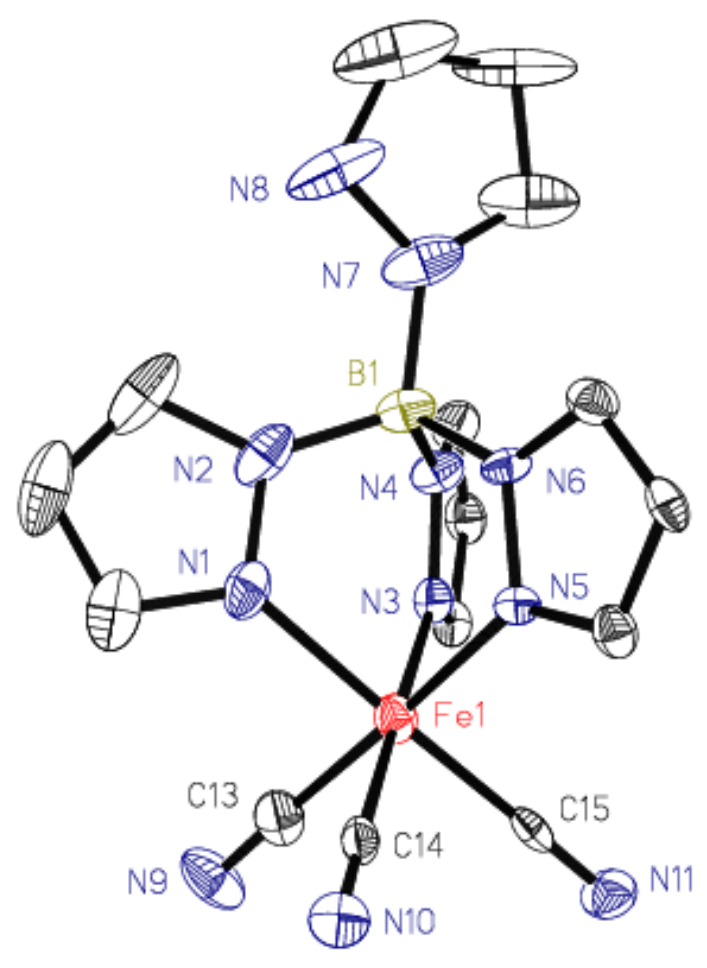

Figure S9. X-ray structure of $\left[\mathrm{NEt}_{4}\right]_{2}\left[(\mathrm{pzTp}) \mathrm{Fe}^{\mathrm{II}}(\mathrm{CN})_{3}\right] \cdot 2 \mathrm{H}_{2} \mathrm{O}$. Thermal ellipsoids are at the $50 \%$ probability level and all cations, lattice solvent, and hydrogen atoms have been eliminated for clarity. 